\title{
The Accuracy of Measurements of Viscosity of Liquids
}

\author{
Robert S. Marvin
}

Institute for Basic Standards, National Bureau of Standards, Washington, D.C. 20234

(July 28, 1971)

\begin{abstract}
Most absolute measurements of viscosity have utilized capillary flow, and required semiempirical corrections amounting to several times their precision and estimated accuracy. The range of values found from these measurements and the possibility of unrecognized systematic errors make it impossible to base a realistic estimate of accuracy on the results of only one type of measurement. The results of two independent absolute measurements involving different types of flow, reported in the two accompanying papers, are summarized here. The estimated accuracy in each case is about 0.1 percent. The two results differ by 0.5 percent. It is suggested that we continue to base the calibration of relative viscometers on the value of 1.002 centipoise $(\mathrm{cP})$ for the viscosity of water at $20{ }^{\circ} \mathrm{C}$ and one atmosphere. This provides a generally accepted base which limits comparability only by the precision of the measurements. However, whenever the true values of viscosity are required the limits of uncertainty including an estimate of systematic error should be taken as no better than \pm 0.25 percent.
\end{abstract}

Key words: Absolute measurement of viscosity; accuracy of viscosity measurement; calibration of viscometers; viscosity; uncertainty of viscosity measurements.

\section{Introduction}

The viscosity (shear viscosity) of a Newtonian liquid is normally measured by a relative technique in an instrument calibrated using a liquid of known viscosity. The common practice is to calibrate viscometers by a step-up technique, using a series of instruments and test fluids, based on the viscosity of water.

For viscosities above 1000 poise $(\mathrm{P})$ or so, the accuracy of such measurements is generally limited by their variability. Such liquids normally have a very high temperature coefficient of viscosity, and problems of adequate temperature control alone make it difficult to attain better than one percent agreement. The agreement between measurements using various standard and accepted types of viscometers $[1,2,3],{ }^{1}$ gives us sound grounds for believing that systematic errors can be kept below this one percent level.

It is quite common and relatively simple to make measurements to within 0.1 percent on ordinary liquids with viscosities below $1 \mathrm{P}$. It is often assumed that this precision also represents the accuracy of such measurements. It appears that any systematic errors associated with the step-up procedure are less than 0.1 percent at least up to viscosities of $1 \mathrm{P}$, although most of the evidence on which this conclusion is based has been obtained with capillary viscometers, leaving a possibility of some unrecognized bias. There remains a question of the accuracy of the value used for the viscosity of the initial calibrating liquid, that is of our absolute measurements of viscosity. This question is the subject of this and the two accompanying papers $[4,5]$.

\section{Systematic Errors in Absolute Measurements}

Nearly all absolute measurements of viscosity have been based on capillary flow because of the high precision attainable. The analysis of capillary measurements is based on the Poiseuille equation,

$$
P_{z} / Q=8 \eta /\left(\pi r^{4}\right),
$$

where $P_{z}$ is the pressure gradient along the capillary in the region where steady flow exists, $Q$ the rate of flow (volume/time), $\eta$ the viscosity, and $r$ the radius of the (circular) capillary tube. In the usual case $P_{z}$ is approximated by $P / l$, where $P$ is the total pressure drop between reservoirs connected by a capillary of length $l$. Two small corrections are normally introduced to correct this approximation. One, the kinetic energy correction, accounts for the pressure increment, proportional to $Q^{2}$, required to accelerate the liquid to the steady (parabolic) velocity profile consistent with eq (1). The other, the Couette correction, accounts for the pressure increment, proportional to $Q$, required to overcome viscous flow resistance in the reservoirs. This can be expressed as a small addition to the length and is normally taken as proportional to the radius of the capillary. The inclusion of these two corrections in (1) yields:

$$
P / Q=8 \eta(l+n r) /\left(\pi r^{4}\right)+m \rho Q /\left(\pi^{2} r^{4}\right)
$$


where $\rho$ is the density and $m$ and $n$ are presumed to be constants for a particular instrument.

The many attempts to derive eq (2), going back to the nineteenth century, are based on assumed flow patterns not exactly realized in practice $[6,7,8,9]$. Different calculations have yielded various values for the "constants" $m$ and $n$, and experimental tests suggest that they differ appreciably for differing velocities of flow, capillary dimensions, and entrance and exit shapes. For square-cut ends, $P / Q$ appears to be independent of $Q$ at low flow rates and linear in $Q$ at higher rates [10]. Trumpet shaped entrances diminish the magnitude of this kinetic energy effect and appear to create flow conditions more compatible with the most convincing theoretical derivations. But even in this case the effective value of $m$ is a pronounced function of Reynolds number, varying from less than 0.1 to 1.0 according to one study [11]. As a rule absolute measurements have been made with square-cut ends on the ground that this configuration permits a more precise determination of $l$, or of $\Delta l$ if two capillaries are employed, than would a more complicated shape.

Pressure drops between taps through a capillary or pipe wall have sometimes been used to eliminate the need for these corrections, but generally in work at high Reynolds numbers rather than in measurements intended for the most accurate determinations of viscosity. Many measurements have taken $m$ and $n$ as empirical constants to be evaluated by varying $Q$ and $l$. The magnitude of these two correction terms varies widely in different measurements; one or both ordinarily influence the final result by several tenths of one percent, and in some cases by several percent. One can certainly question whether corrections of this magnitude with a somewhat nebulous theoretical basis can justify an assumption that any associated systematic error is below 0.1 percent.

Other possible sources of systematic error, which may be even more significant in some cases, have influenced various absolute measurements in capillary instruments. One arises from the normal use of capillaries with diameters of a millimeter or less, and the problem of obtaining capillaries of this size with uniform bores. The average radius of such capillaries can be measured with adequate accuracy, and a reasonable correction for any small average ellipticity or for a small uniform taper made. However, any irregular variation in radius along the length would seem bound to result in radial flow whose influence cannot be calculated. The information available on this type of variatıon in the tubes used in earlier determinations, quite limited in many cases, indicates that irregular variations in radius of at least 1 percent are common.

Other methods of absolute measurement are subject to difficulties analogous to those above. In nearly all cases some type of end effect or wall effect which cannot be calculated exactly is present. Some variation in the assumed geometrical shape is present, causing some variation from the flow assumed and hence a possible systematic error in the results. With many methods it is difficult to attain the precision required for an overall uncertainty of 0.1 percent.

\section{Survey of Previous Measurements}

Most absolute measurements of viscosity which have aimed at an accuracy of better than 1 percent have been made on water. Most of the early measurements employed capillary flow. Bingham and Jackson [12] arrived at a value of $1.005 \mathrm{cP}$ for the viscosity of water at $20{ }^{\circ} \mathrm{C}$, based on their evaluation of all the available measurements (at several temperatures) they considered justified in including as valid absolute measurements. These ranged from the work of Poiseuille in 1840-46 to that of Washburn and Williams in 1913. Dorsey, considering the same set of values, arrived at a value of 1.009 on which he based his tabulations for the International Critical Tables [13]. In an attempt to resolve this discrepancy, Bingham [14] presented the conclusions of a group which had reexamined the same measurements, stating: "No research has been sufficiently complete so that all of the needed corrections can be estimated with sufficient accuracy."

The value for the viscosity of water now generally accepted, $1.002 \mathrm{cP}$ at $20{ }^{\circ} \mathrm{C}$ and $1 \mathrm{~atm}$, was reported by Swindells, Coe, and Godfrey (SCG) [15] in 1952 based on work which extended over a period of some twenty years. They used four capillaries with squarecut ends, two with lengths differing by approximately a factor of two for each of two radii differing by about 20 percent.

In this work the Couette correction was negligible, but the kinetic energy correction for individual measurements varied from 0.3 to 5 percent as $Q$ was varied to give Reynolds numbers ranging from 100 to 650 . Their capillaries were more uniform than most used in earlier work, with a maximum variation in diameter of about \pm 0.5 percent and about half that variation in the mean diameters at various positions along the tubes. They established a mean $Q$ with a piston driven by a synchronous motor through a gear train, and measured the pressure in input and output reservoirs with mercury manometers. Later attempts to use this same injector with a pressure measuring device having a much shorter response time disclosed a high frequency fluctuation in $Q$, representing an additional deviation from the assumed flow.

The "accuracy" of \pm 0.03 percent estimated by SCG represents the standard deviation of the mean of values calculated from the intercepts of linear relations between $P / Q$ and $Q$ for the four tubes, treated both individually and in pairs. Deviations of individual measurements from the calculated lines did not exceed 0.04 percent and appear random. Uncertainties in values of the average radii, lengths, mean rates of flow, and temperature were ignorable, but of course no allowance could be estimated for the type of possible systematic error discussed in the preceding section.

Only a few attempts at an absolute measurement have been made since the work of SCG. Roscoe and Bainbridge [16] measured the decrement of a glass sphere filled with water and suspended from a torsion wire. They reported a value of $1.0025 \mathrm{cP}$ with a computed standard error of $0.0005 \mathrm{cP}$. This error was 
estimated from the variability found in measuring the various quantities involved. The magnitude of several possible systematic errors cannot be estimated. The polar and equatorial diameters of their sphere differed by 0.3 percent, which could cause some deviation from the assumed flow. A three percent correction was required for the effect of air damping. This was a theoretical estimate with a correction based on the differences between measurements with air and with a rigid gel in the sphere and theoretical estimates for those two conditions.

G. A. Maliarov [17] reported a value of 1.0035 with an uncertainty of \pm 0.1 percent. This was based on the differences between the pressure drops across two capillaries connected in series through a central reservoir. Unexplained variations in the reported replicate pressure measurements across each individual capillary were more than ten times the variations found for the differences between the pressure drops across the individual capillaries in series. For two of these pairs the rates of flow reported covered a range sufficient to make an estimate of the corrections required to calculate viscosity values from pressure drops across individual capillaries. This calculation yields values varying from 1.002 to 1.014 , a difference which appears inconsistent with the estimated uncertainty of 0.1 percent.

Kawata, Kurase, and Yoshida [18] measured the viscosity of a $1.89 \mathrm{P}$ hydrocarbon liquid using an absolute capillary technique essentially the same as that employed by SCG. Their Reynolds numbers were 0.14 and below and no kinetic energy correction was needed, but they did require a Couette correction ranging from 0.3 to 0.5 percent. This was determined empirically from measurements with two capillaries, yielding values of $n$ in eq (2) which ranged from 0.79 to 0.88 for different flow rates. This measurement yielded values consistent with a viscosity of water at $20{ }^{\circ} \mathrm{C}$ of $1.0016 \mathrm{cP}$ as determined by relative capillary measurements. These were equivalent to the relative measurements used in this work and discussed in section 5. Even if the measurement of viscosity ratios can be considered free of systematic error up to this range the magnitude and uncertain nature of the Couette correction required leave a question as to whether this agreement is enough to confirm the value of $1.002 \mathrm{cP}$ to 0.1 percent. It should be noted that the authors did not present these results as a check of the viscosity of water, but rather of the adequacy of the customary step-up calibration procedure.

\section{Objectives and Outline of This Study}

We conclude that existing absolute measurements are inadequate for estimating the accuracy of our values of viscosity within limits comparable to their precision. The stated uncertainties of the best measurements are based on the precision attained. Some possible systematic errors associated with deviations from the assumed flow cannot be evaluated. Though there is good agreement of the Roscow and Bainbridge measurement with the SCG value, both involve indirect or empirical corrections uncomfortably large compared to the accuracy claimed.

In most cases where a comparison of viscosity measurements to 0.1 percent is sought, relative measures referred to a common base are sufficient. The "true" value of viscosity is seldom needed with an accuracy of better than 1 percent. However, we certainly cannot claim to understand our measurement process until we have established a reliable estimate of the limits of systematic errors.

The only way of arriving at such an estimate is by comparison of absolute measurements utilizing different types of flow. To reduce the uncertainty associated with the comparison of earlier measurements the geometry should be realized more closely than in previous work, and this realization should be confirmed by independent checks. Any corrections required should be based as nearly as possible on direct measurements. And of course the precision must be commensurate with the accuracy of the final comparison. Even though we might not be certain we had identified all possible systematic errors in either method, the difference found between measurements meeting these criteria should indicate the probable magnitude of any unrecognized systematic errors.

The first of the two methods we selected was based on a measurement of the period of oscillation of a sphere filled with liquid and supported by a torsion wire [4]. If the liquid is Newtonian, its viscosity may be determined from either the period or decrement. Since period can be measured with much greater accuracy, our instrument was designed to maximize the sensitivity of period, rather than decrement, in the design range. There are no end effects to disturb the flow of liquid within a sphere. The two basic problems we can identify in this method are producing a sphere with uniform internal diameter and the possibility of a secondary flow caused by inertial forces.

The second method used a modified capillary flow technique [5]. A much more uniform channel than any previously employed was obtained by forming it from two accurate cylinders and an optical flat, yielding a pipe with a triangular cross section with one side a straight line and the other two circular arcs. In order to eliminate end effects, pressure drops were measured at taps through the pipe wall. Four taps with varying spacing, and flows in both directions at several rates were employed in an attempt to detect any possible effect of a perturbation of flow at the taps.

Since this was planned as an experiment to assess systematic errors rather than as another measurement of the viscosity of water, we used di(2-ethylhexyl) sebacate as a test fluid. It has a viscosity about twenty times that of water, and a much lower vapor pressure and surface tension. We used a commercial grade purified by molecular distillation, Octoil $\mathrm{S},{ }^{2}$ for the sphere measurements. For the channel meas-

2 This material, as presently available, is apparently not reproducible enough to use in lieu of water in the calibration of viscometers. The viscosity of three batches, provided through the courtesy of Dr. B. B. Dayton of the Vacuum Division of the Bendix Corporation, yielded values varying by two percent. 
urements, which required much larger quantities of fluid, we used Plexol 201, a less expensive grade of the same chemical. The correction for the small difference in viscosity of these two test fluids, based on relative measurements, did not introduce any significant uncertainty into our comparison of the two results as will be shown later.

For convenience in relating our results to other work, we have referred all measurements to the viscosity of water at $20{ }^{\circ} \mathrm{C}$ by comparing the values measured by the absolute techniques with those measured in a pair of Cannon Master intruments calibrated in the usual fashion in terms of water. It would be entirely equivalent, so far as the conclusions we shall draw, to express our results in terms of the viscosity at $25^{\circ} \mathrm{C}$ of either of the samples of di(2-ethylhexyl) sebacate used.

\section{Results and Discussion}

Our measurements of viscosity in the oscillating sphere intrument gave values consistent with a viscosity of water at $20{ }^{\circ} \mathrm{C}$ of $1.006 \mathrm{cP}$. The sum of the magnitudes of all systematic errors that could be identified came to 0.07 percent. The major contributions to this figure came from an uncertainty in the radius of the sphere of 0.01 percent, and an uncertainty in the constant of the torsion wire of 0.015 percent. Some radial flow, expected at large amplitudes, was presumed to account for a slight dependence of the observed period on amplitude which could be seen only at amplitudes much larger than those used in these measurements.

The temperature of the sphere and liquid was known and constant to $\pm 0.005{ }^{\circ} \mathrm{C}$; that of the torsion wire to $\pm 0.05{ }^{\circ} \mathrm{C}$. Variations within these limits will cause undetected changes in the viscosity of the liquid and in the spring constant and hence a variability in the final measurements. Repeated measurements of the moment of inertia of the empty sphere showed a spread of \pm 0.01 percent, apparently due to a slight variability in the condensed or adsorbed material on the sphere wall after cleaning and subjecting to a vacuum. These three factors can be combined to predict the variability in the measured values of viscosity either by summing the absolute values to yield 0.1 percent or by taking the square root of the sum of squares to yield 0.066 percent. Eighteen observations were included within limits of \pm 0.069 percent about the average, with a coefficient of variation of 0.03 percent.

The channel flow measurements yielded a value compatible with a viscosity of water at $20{ }^{\circ} \mathrm{C}$ of 1.001 $\mathrm{cP}$. Here the major contributions to the estimated systematic error were \pm 0.04 percent from uncertainties in the geometry of the channel and \pm 0.06 percent in the pressure measurements. The sum of the magnitudes of all contributions was 0.13 percent. The coefficient of variation of the pressure measurements was 0.02 percent; all measurements were included within the limits of \pm 0.03 percent about the mean. As in other pipe flow measurements, no estimate of the effect of possible radial flow can be made. However, the deviations from the assumed shape here were much less than the variation in the internal radii of any of the capillaries used in previous measurements.

The sphere measurements were made on Octoil S both saturated with air and air-free and under various pressures from $1 \mathrm{~atm}$ down to less than $0.2 \mathrm{~mm} \mathrm{Hg}$. The simplest consistent presentation of all these results and their comparison with measurements on Plexol 201 in the channel is made by relating them all to the viscosity of water at $20{ }^{\circ} \mathrm{C}$ using conventional relative capillary viscometers. However, our final comparison of the results of these two measurements does not rely on the accuracy of the instrument constants of our relative instruments.

All the relative viscosity measurements were made in one pair of Cannon Master viscometers with constants of about $0.04 \mathrm{cSt} / \mathrm{s}$. The viscosities of our Octoil $\mathrm{S}$ and Plexol 201 differed by slightly less than one percent. The kinetic energy correction was completely negligible, regardless of any reasonable value assigned $m$ in eq (2). Our instruments were filled at $25 \pm 1{ }^{\circ} \mathrm{C}$, and the nominal $25^{\circ}$ runs made at $25.000 \pm 0.001{ }^{\circ} \mathrm{C}$. The surface tensions of hydrocarbons of this type do not differ enough to require a correction when comparing flow times; a difference of ten percent would require a correction to the ratio of flow times of 0.01 percent.

Thus the true kinematic viscosities of these two liquids should be accurately proportional to the flow times. Any uncertainty in the value of the proportionality constant can be avoided, for the purpose of co!nparing our two absolute measurements, by comparing ratios of the two absolute viscosities as measured in the sphere and channel with the ratio of flow times for the same two liquids in one of the relative viscometers.

A number of measurements were made on each of our liquids in the two relative viscometers. Typical results are shown in table 1 . The two samples of Octoil $\mathrm{S}$ were taken about two years apart, once near the beginning and once near the end of an extended series of measurements in the sphere. The samples of Plexol 201 were taken from the channel apparatus before each

TABle 1. Flow times for Octoil $S$ and Piexol 201 in relative viscometers at $25^{\circ} \mathrm{C}$

\begin{tabular}{|c|c|c|c|c|}
\hline & \multicolumn{2}{|c|}{ Viscometer M-204 } & \multicolumn{2}{|c|}{ Viscometer M-205 } \\
\hline & Octoil S & Plexol 201 & Octoil S & Plexol 201 \\
\hline \multirow[t]{3}{*}{ 1st Sample } & 481.13 & 485.80 & 486.24 & 490.70 \\
\hline & 481.06 & 485.82 & 486.14 & 490.76 \\
\hline & 481.01 & 485.76 & 486.11 & $\underline{490.61}$ \\
\hline Av. & 481.067 & $\overline{485.793}$ & $\overline{486.163}$ & 490.690 \\
\hline \multirow[t]{3}{*}{ 2nd Sample } & 481.05 & 485.84 & 485.93 & 490.50 \\
\hline & 481.04 & 485.73 & 486.05 & 490.50 \\
\hline & $\begin{array}{l}481.22 \\
481.03 \\
\end{array}$ & 485.81 & $\begin{array}{l}486.23 \\
486.21\end{array}$ & 490.42 \\
\hline Av. & $\overline{481.085}$ & $\overline{485.793}$ & $\overline{486.105}$ & $\overline{490.473}$ \\
\hline Av., 2 Samples & 481.077 & 485.793 & 486.130 & 490.582 \\
\hline & 0.073 & 0.041 & 0.112 & 0.132 \\
\hline \multicolumn{5}{|l|}{ Ratio, Plexol/ } \\
\hline Av. Ratio & \multicolumn{2}{|c|}{1.0095} & \multicolumn{2}{|c|}{1.0092} \\
\hline
\end{tabular}


of two runs on successive days. The estimates of standard deviation of the inidividual flow measurements shown, corresponding to coefficients of variation of 0.027 percent or less, are consistent with those normally found with instruments of this type.

These flow times were measured at atmospheric pressure with air-saturated samples. The average ratio in the two instruments is 1.0095 . The ratio of the viscosities of the two liquids, again both air-saturated and at atmospheric pressure, as measured in the channel and sphere instruments is 1.0043. This difference of 0.5 percent is ten times the coefficient of variation of any of the measurements involved and about five times our estimate of the maximum systematic error for either of the absolute measurements. It is apparent that some unrecognized systematic error is present in one or both of the absolute measurements.

The comparison of these with earlier measurements can be done only through our measurements of the ratios of the viscosities of our test liquids to that of water by use of our relative viscometers. This involves measurements of the ratios of flow times of water and one intermediate liquid in one set of instruments, of the first and a second intermediate liquid in a second set, and of the second intermediate and our samples in a third set. Thus three ratios of flow times are involved. The first, involving water, required a correction of 0.12 percent because of the large difference in surface tensions. The others involved corrections of about 0.01 percent. The kinetic energy corrections were 0.015 percent or less. The Reynolds numbers involved ranged from 0.3 to 22 .

Since we have not identified the systematic errors in the absolute measurements, we cannot arbitrarily rule out the possibility of some unsuspected systematic error in our measurement of viscosity ratios. However, it is difficult to believe that any such error could exceed 0.1 percent, the maximum correction involved in obtaining the ratios between the viscosities of our test liquids and water. If we accept this conclusion we would say that our channel flow measurement corresponds to a value for the viscosity of water marginally lower than the SCG value, and the sphere measurement one significantly higher. Also our two measurements correspond to values which include most of the earlier measurements on water and all of those since the work of SCG.

\section{Conclusion}

From the results of our measurements and the indirect comparison of these with earlier measurements, we conclude that the best estimate of the contribution of systematic errors to the total uncertainty associated with values of the viscosity of liquids should be taken as \pm 0.25 percent. This is 2.5 times the quantity now generally assumed.

These results do not suggest a change in the value of $1.002 \mathrm{cP}$ now generally used as the viscosity of water at $20^{\circ} \mathrm{C}$ in the calibration of relative viscometers. We have no grounds for believing either of our results is more likely to be correct than the other. And their average, which differs from either measurement by 2.5 times its expected uncertainty, is unlikely to be correct.

Most users who desire precise viscosity values require only agreement between measurements in different laboratories, achieved by referring their relative measurements to a common base. Such comparisons need not include the additional uncertainty associated with the absolute measurements and there is no present justification to change the accepted base. In cases where the true value of viscosity is important, a value based on $1.002 \mathrm{cP}$ for the viscosity of water at $20^{\circ} \mathrm{C}$ seems as good a choice as any, but we believe this value should be assigned an uncertainty of \pm 0.25 percent.

This work extended over a number of years, and several individuals made contributions which we are pleased to acknowledge. James F. Swindells participated in many of our early discussions and his knowledge of earlier work (both his own and that of others) and of many far from obvious pitfalls and difficulties was most helpful. Herbert Goldstein, now with the Patent Office, carried out the early development work for the pipe flow measurement. His work uncovered fatal defects in several approaches we had all originally expected would be satisfactory. Theodore R. Young suggested the geometry of the pipe finally used and made a number of valuable suggestions about its assembly. Professor Joseph Kestin of Brown University and Dr. R. E. Manning of the Cannon Instrument Company have given us very helpful advise and suggestions concerning various aspects of the experimental work and interpretation of the results. Dr. Kawata provided us with some additional, unpublished, information about the absolute measurements in the Kawata, Kurase, and Yoshida work [18].

\section{References}

[1] Barr, G., A Monograph of Viscosity, (Oxford University Press, London, 1931)

[2] Merrington, A. C., Viscometry (Edward Arnold \& Co., London, 1949).

[3] van Wazer, J. R., Lyons, J. W., Kim, K. Y., and Colwell, R. E., Viscosity and Flow Measurement, (Interscience, New York, 1963).

[4] White, H. S., and Kearsley, E. A., An absolute determination of viscosity using a torsional pendulum, J. Res. Nat. Bur. Stand. (U.S.), 75A (Phys, and Chem.) No. 6, 541-551. (Nov.-Dec. 1971).

[5] Penn, R. W., and Kearsley, E. A., An absolute determination of viscosity using channel flow, J. Res. Nat. Bur. Stand. (U.S.), 75A (Phys. and Chem.), No. 6, 553-560. (Nov.-Dec. 1971).

[6] Barr, G., loc. cit., pp. 12-35.

[7] Goldstein, S., Modern Developments in Fluid Dynamics, Vol. 1, pp. 297-308, (Oxford University Press, London, 1938). 
[8] Weissberg, H. L., End corrections for slow viscous flow through long tubes, Phys. Fluids 5, No. 9, 1033-1036 (Sept. 1962).

[9] Christiansen, E. B., and Lemmon, H. E., Entrance region flow A.I.Ch.E. J. 11, No. 6, 995-999, (Nov. 1965).

[10] Swindells, J. F., Hardy, R. C., and Cottington, R. L., Precise measurements with Bingham viscometers and Cannon Master viscometers, J. Res. Nat. Bur. Stand. (U.S.), 52, No. 3, 105-120, (March 1954) RP 2479.

[11] Cannon, M. R., Manning, R. E., and Bell, J. D., Viscosity measurement, The kinetic energy correction and a new viscometer, Anal. Chem. 32, No. 3, 355-358 (March 1960); Einfeldt, J., Zur Kapillarviskosimetrie verdünnter Lösungen, II. Bestimmung der Abhängigkeit der kinetischen Energiekorrektur von der Ausflusszeit, Rheologica Acta 9 (3), 429-434, (1970).

[12] Bingham, E. C., and Jackson, R. F., Standard substances for the calibration of viscometers, Nat. Bur. Stand. (U.S.) Bull. 14, (1919), pp. 59-86.

[13] Dorsey, N. E., Viscosity of water, sulfuric acid, liquid carbon dioxide and certain organic liquids, Int. Crit. Tables $\mathbf{5}$, Ed. E. W. Washburn, p. 10, (McGraw-Hill, New York,
1929); Properties of Ordinary Water Substance, pp. 182-184, (Reinhold Pub. Co., New York, 1940).

[14] Bingham, E. C., Notes on the fluidity of water at $20^{\circ} \mathrm{C}, \mathrm{J}$. Rheology 2, No. 4, pp. 403-423, (Oct. 1931).

[15] Swindells, J. F., Coe, J. R., Jr., and Godfrey, T. B., Absolute viscosity of water at $20^{\circ} \mathrm{C}$, J. Res. Nat. Bur. Stand. (U.S.), 48, No. 1, pp. 1-31, (Jan. 1952) RP 2279.

[16] Roscoe, R. and Bainbridge, W., Viscosity determination by the oscillating vessel method, II. The viscosity of water at $20{ }^{\circ}$ C. Proc. Physical Society 72, Part 4, pp. 585-595, (Oct. 1958).

[17] Mailiarov, G. A., Absolute viscosity of water at a temperature of $20{ }^{\circ} \mathrm{C}$, Trudy Vses. Nauchn-Issled, Inst. Metrl. (Proc. of the All-Union Sci. Res. Inst. of Metrology), No. 37(97), pp. 125-140 (1959). (In Russian.)

[18] Kawata, M., Kurase, K., and Yoshida, K., Realization of a viscosity standard, Proc. Fifth Int. Congress on Rheology, Vol. 1, Ed. S. Onogi pp. 453-472 (Univ. of Tokyo Press, 1969).

(Paper 75A6-684) 